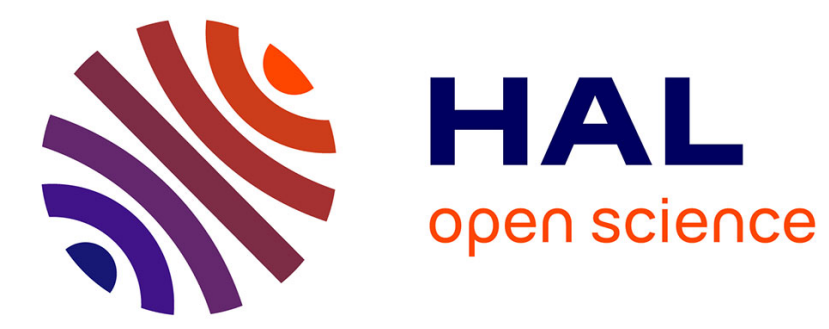

\title{
SIMULATIONS NUMÉRIQUES DE LA PROPAGATION DU SON DANS L'ATMOSPHÈRE
}

\author{
D. Juve
}

\section{To cite this version:}

D. Juve. SIMULATIONS NUMÉRIQUES DE LA PROPAGATION DU SON DANS L'ATMOSPHÈRE. Journal de Physique IV Proceedings, 1992, 02 (C1), pp.C1-537-C1-544. 10.1051/jp4:19921116 . jpa-00251071

\section{HAL Id: jpa-00251071 https://hal.science/jpa-00251071}

Submitted on 1 Jan 1992

HAL is a multi-disciplinary open access archive for the deposit and dissemination of scientific research documents, whether they are published or not. The documents may come from teaching and research institutions in France or abroad, or from public or private research centers.
L'archive ouverte pluridisciplinaire HAL, est destinée au dépôt et à la diffusion de documents scientifiques de niveau recherche, publiés ou non, émanant des établissements d'enseignement et de recherche français ou étrangers, des laboratoires publics ou privés. 


\title{
SIMULATIONS NUMERIQUES DE LA PROPAGATION DU SON DANS L'ATMOSPHÈRE
}

\section{JUVE}

Laboratoire de Mécanique des Fluides et d'Acoustique, CNRS URA-263, Ecole Centrale de Lyon, BP. 163, F-69131 Ecully cedex, France

\begin{abstract}
Sound propagation in the lower atmosphere is greatly influenced by mean velocity and temperature gradients and by turbulent fluctuations. After a short description of the relevant meteorological parameters we give a review of the main numerical techniques used to take into account these deterministic as well as random perturbations. Various illustrations will be provided at the conference
\end{abstract}

\section{INTRODUCTION}

La propagation du son au voisinage du sol est influencée par de nombreux facteurs comme la nature du terrain, le relief, les profils moyens de température et de vent, la turbulence. Ces différents facteurs ne jouent pas de façon indépendante ; par exemple la topographie influence les profils de vent et la turbulence ; l'effet de la nature du terrain sera plus ou moins important selon que le vent rabattra ou non l'énergie acoustique vers le sol. A terme il faudra donc disposer de modélisations permettant la prise en compte de tous ces paramètres. Cependant la complexité des phénomènes mis en jeu fait que ce but est encore loin d'être atteint, et qu'il est instructif de considérer les différents facteurs sinon indépendamment les uns des autres, du moins par tous simultanément. Dans cet article, le sol sera ainsi pris en compte sous la forme d'une condition limite simple [1], de type impédance (modèle de Delany \& Bazley) voire de rigidité parfaite, notre intérêt se portant spécifiquement sur le rôle des inhomogénéités du milieu de propagation.

Après avoir brièvement rappelé les conditions météorologiques typiques de la basse atmosphère, nous décrirons les méthodes numériques qui nous semblent les plus appropriées pour résoudre les problèmes de propagation déterministes et aléatoires. Des illustrations des effets les plus caractéristiques que l'on peut observer (réfraction, convection, fluctuations de niveau) seront présentées lors de la conférence.

\section{QUELQUES ELEMENTS DE METEOROLOGIE}

Les premières centaines de mètres au-dessus du sol sont caractérisées par la présence d'une couche limite atmosphérique où coexistent gradients moyens de vent et de température et fluctuations turbulentes. Dans une région au relief peu accidenté, le vent possède une composante horizontale prépondérante ne dépendant que de l'altitude qui peut être souvent modélisée par un profil logarithmique :

$$
\frac{V(z)}{V_{0}}=K_{v} \log \left(z / z_{0}\right) \quad z>z_{0}
$$


Le paramètre $z_{o}$ est caractéristique de la rugosité de la surface et peut être considéré comme la hauteur d'un obstacle type. Le tableau I donne quelques valeurs de cette hauteur de rugosité pour différents sites. La constante $K_{v}$ dépend pour sa part de la rugosité et de la vitesse du vent en altitude, et $V_{0}$ désigne la vitesse de frottement.

L'évolution verticale de la température est souvent donnée par une formule du même type :

$$
T(z)=T_{0}-K_{T} \log \left(z / z_{0}\right) \quad z>z_{0}
$$

où $\mathrm{T}_{\mathrm{o}}$ désigne la température "au sol" (pour $\mathrm{z}<\mathrm{z}_{\mathrm{o}}$ ) et $\mathrm{K}_{\mathrm{T}}$ est un paramètre dont le signe dépend des conditions météorologiques. Le signe plus correspond à la décroissance "normale" de la température avec l'altitude que l'on observe à une échelle plus globale; le signe moins est associé au phénomène d'inversion provoqué par le refroidissement du sol par rayonnement lors d'une nuit claire. Ces deux comportements sont illustrés sur la figure 1.

En plus de ces gradients moyens existent également des fluctuations aléatoires de vent et de température. Ces fluctuations turbulentes mettent en jeu des structures de différentes tailles entre une échelle externe (les grands tourbillons transférant l'énergie du mouvement moyen vers la turbulence) et une échelle interne (imposée par la dissipation visqueuse). La caractérisation de la turbulence repose sur la mesure du spectre énergétique des fluctuations et c'est bien la grandeur nécessaire pour l'application des théories de la propagation en milieu turbulent. En pratique on se contente souvent de la mesure de deux quantités : l'intensité de la turbulence, c'est-à-dire la valeur efficace des fluctuations thermiques et cinématiques, et l'échelle de corrélation de ces fluctuations qui caractérise leur cohérence spatiale. Le spectre est ensuite représenté par un modèle, de von Karman au mieux, limité souvent à la zone inertielle de Kolmogorov, voire simplement gaussien. Le tableau II donne quelques valeurs d'intensité de turbulence et de longueur de corrélation relevées lors d'expériences de propagation dans l'atmosphère. On doit noter à ce propos que l'un des problèmes essentiels des mesures in situ réside dans le caractère très lacunaire des informations météorologiques relevées lors des essais, ce qui rend délicate ou discutable l'interprétation des résultats. Si elles demeurent indispensables, il est clair que des essais complémentaires en soufflerie et des simulations numériques sont très utiles pour dégager l'influence d'un facteur particulier pris individuellement.

\section{EQUATIONS DE PROPAGATION}

Dans la grande majorité des calculs de propagation atmosphérique l'équation utilisée au départ est l'équation de Helmholtz à indice variable :

$$
\Delta \mathrm{p}+\mathrm{k}_{\mathrm{o}}^{2} \mathrm{n}^{2} \mathrm{p}=0 ; \mathrm{n}=\frac{\mathrm{c}_{0}}{\mathrm{c}} ; \mathrm{k}_{\mathrm{o}}=\frac{\omega}{\mathrm{c}_{\mathrm{o}}}
$$

où $c_{\mathrm{o}}$ désigne une valeur moyenne de la célérité locale $\mathrm{c}$.

Il faut cependant souligner que l'équation (1) n'est qu'une approximation de l'équation complète pour un milieu inhomogène au repos :

$$
\Delta p+k_{o}^{2} n^{2} p+\frac{1}{\rho} \overrightarrow{\operatorname{grad}} \rho \overrightarrow{\operatorname{grad}} p=0
$$

qui ne se réduit à (1) que dans une approximation de hautes fréquences. En acoustique aérienne, pour des fréquences de l'ordre de la centaine de Hertz, l'influence des gradients de masse volumique peut être aussi grande que celle de la variation de célérité du son et l'utilisation de l'équation (2) est donc souhaitable. 
D'autre part lorsque le milieu est en mouvement (vent) d'autres effets complexes se manifestent. En particulier il existe un phénomène de convection de l'onde par la vitesse locale de l'écoulement ; en se limitant au ler ordre par rapport au nombre de Mach et en négligeant l'effet des gradients on obtient :

$$
\Delta p+k_{0}^{2} p+2 i k_{0} M \frac{\partial p}{\partial r}=0
$$

où $\mathrm{M}=\frac{\mathrm{V}}{\mathrm{c}_{\mathrm{o}}}$, la vitesse étant supposée horizontale (coordonnée $\mathrm{r}$ ). Le nouveau terme en $\partial \mathrm{p} / \partial \mathrm{r}$ n'est en général pas correctement pris en compte, sauf dans la théorie géométrique : on se ramène l'étude à une propagation dans un milieu inhomogène en introduisant un "indice équivalent" $n=\frac{c_{o}}{c_{\text {eff }}}$ où la vitesse effective $c_{\text {eff }}$ est donnée par

$$
c_{\text {eff }}=c_{o}+V \cos \theta
$$

$\theta$ désignant l'angle formé par la direction du vent et la direction initiale de propagation. Une analyse détaillée des erreurs introduites par ce type d'approximation en acoustique aérienne reste à effectuer.

La prise en compte des fluctuations turbulentes du milieu se fait classiquement en considérant que l'indice $n$ est une variable aléatoire dans l'équation (1) (ou dans son approximation parabolique) et en effectuant une moyenne d'ensemble pour calculer les moments successifs du champ de pression : partie cohérente de l'onde, corrélation, fluctuations d'intensité. Malheureusement, le système d'équations que l'on obtient n'est pas "fermé" ; il est nécessaire d'introduire une hypothèse a priori sur la corrélation entre la fluctuation d'indice et l'onde acoustique (hypothèse de corrélation microscopique selon la direction moyenne de propagation, [3]). Cette hypothèse donne de bons résultats pour les deux premiers moments mais semble plus discutable pour le calcul des fluctuations d'intensité. Récemment une approche différente a été développée pour s'affranchir de l'hypothèse de corrélation microscopique ; elle consiste à résoudre une équation d'onde non moyennée (déterministe) pour chaque réalisation du champ turbulent et à n'effectuer les moyennes statistiques que sur le résultat de ces calculs sur un ensemble de réalisations [4], [5], [6]. Cette nouvelle approche est certes coûteuse en temps de calcul, mais elle semble très prometteuse et peut s'accommoder assez facilement de conditions moyennes inhomogènes : existence de gradients thermiques, présence d'un sol...

\section{METHODES DE RESOLUTION}

Les méthodes de résolution sont relativement nombreuses; elles proviennent d'autres domaines de la physique (optique, sismique, acoustique sous-marine) et ont donc été développées à l'origine pour un milieu au repos. Nous citerons quatre de ces méthodes qui nous semblent à des titres divers les plus adaptées pour traiter de la propagation atmosphérique.

\section{Méthode de la fonction de Green (Fast Field Program)}

Cette méthode repose sur l'hypothèse d'un milieu bidimensionnel stratifié, ce qui limite considérablement son usage dans la pratique. Cependant elle est exacte (aux erreurs numériques près) ce qui en fait un passage quasi-obligé pour la comparaison avec les résultats issus de méthodes approchées [7]. L'idée fondamentale est d'utiliser une transformation de Fourier-Bessel selon la verticale :

$$
p(r, z, \omega)=A \int_{0}^{\infty} G\left(z, z_{S}, K, \omega\right) J_{0}(K r) K d K
$$

$r$ et $z$ sont les coordonnées horizontale et verticale, $z_{s}$ l'altitude de la source, $J_{o}$ la fonction de Bessel de lère espèce d'ordre 0 et $\mathrm{K}$ est un nombre d'onde horizontal. $\mathrm{G}$ désigne une fonction de Green "verticale". 
Le Fast Field Program emploie ensuite un certain nombre d'approximations telles que : développement asymptotique de $\mathrm{J}_{\mathrm{o}}(\mathrm{K} \mathrm{r}$ ), approximation du profil réel de célérité par des fonctions simples ( $\mathrm{c}(\mathrm{z}$ ) constant ou à gradient constant par couche) de façon à calculer explicitement $G$, enfin échantillonnage de $\mathrm{K}$ et de $\mathrm{r}$ permettant l'utilisation d'un algorithme de TFR. Le contrôle des diverses erreurs numériques n'est pas trivial, notamment pour ce qui est de l'approximation du profil de célérité ; ces problèmes sont discutés dans les références [8] et [9].

\section{Approximation géométrioue}

Il s'agit du traditionnel tracé des rayons, pour lequel on construit une solution asymptotique (pour $\omega \rightarrow \infty)$ de l'équation de Helmholtz ou plus généralement des équations linéarisées de la mécanique des fluides [10]. Les rayons sont donnés par la résolution du système différentiel suivant :

$$
\begin{aligned}
& \frac{d x_{i}}{d s}=\frac{V_{i}}{c}+\frac{k_{i}}{k} \\
& \frac{d k_{i}}{d s}=-k \frac{1}{c} \frac{\partial c}{\partial x_{i}}-\frac{1}{c} k_{j} \frac{\partial V_{j}}{\partial x_{i}}
\end{aligned}
$$

$x_{i}$ désignant la position d'un point sur le rayon, $s$ l'abscisse curviligne et $\vec{k}$ le vecteur d'onde local, orthogonal aux surfaces équiphases. Un système complémentaire permet de calculer l'évolution de la section droite d'un tube de rayons infinitesimal, d'où on déduit l'amplitude du champ de pression. L'un des résultats essentiels de la théorie géométrique est la mise en évidence de la convection des surfaces d'onde par l'écoulement qui rend le milieu anisotrope : la vitesse de groupe (propagation de l'énergie) n'est plus colinéaire à $\vec{K}$.

Les avantages et les inconvénients de la méthode géométrique sont bien connus. D'un côté, simplicité (relative) du calcul des rayons, possibilité de prise en compte de profils quelconques de célérité et de vent, d'une topographie complexe et d'effets tridimensionnels. De l'autre, difficulté du calcul des niveaux (détermination des rayons propres) et surtout absence de tout effet de diffraction : perte de validité de l'approximation dans les zones d'ombre et les régions de caustiques.

\section{Approximations paraxiales}

Dans ce type d'approche on profite de l'existence d'une direction privilégiée (l'horizontale) pour construire une équation qui incorpore l'essentiel des effets de diffraction et qui se prête à une résolution numérique efficace. On pose :

$$
\begin{aligned}
& p(r, z)=\psi(r, z) \varphi(r) \\
& \varphi(r)=\frac{e^{i k_{0} r}}{r^{n}} ; n=0,1 / 2 \text { ou } 1
\end{aligned}
$$

$\psi(r, z)$ représente l'enveloppe du signal, dont les variations selon $r$ sont lentes par rapport à celles de $\varphi(r)$. Une analyse d'ordres de grandeur conduit à l'équation parabolique standard [11] :

$$
2 i k_{0} \frac{\partial \psi}{\partial r}+\frac{\partial^{2} \psi}{\partial z^{2}}+k_{o}^{2}\left(n^{2}-1\right) \psi=0
$$

Cette équation peut être résolue par TFR ou par différences finies en avançant selon $r$ à partir de la donnée d'un front initial $\psi\left(r_{0}, z\right)$. La limitation essentielle de l'E.P.S. concerne son domaine angulaire de validité relativement réduit ( $\pm 15^{\circ}$ par rapport à l'horizontale). Cependant des équations grands angles ont été développées, en sismique [12] ou en acoustique sous-marine [13], qui remédient largement à ce défaut (validité sur $\pm 45^{\circ}$ ). 
Les avantages de la méthode sont : une bonne prise en compte de la diffraction, des temps de calcul raisonnables du moins pour les basses fréquences (maillage en $\lambda / 6$ selon la verticale), la possibilité de traiter des problèmes tridimensionnels.

Parmi les inconvénients, citons la non prise en compte de l'énergie rétrodiffusée, des difficultés pour calculer le champ dans les zones d'ombre juste derrière un obstacle (limitations angulaires) et le fait que le calcul est global, donc ne permet pas la dissociation des contributions d'un trajet direct et d'un trajet réfléchi.

\section{Sommation de faisceaux gaussiens}

Il s'agit là de la méthode la plus récente qui tente de cumuler les avantages de la théorie géométrique et de l'approximation parabolique. On donne une épaisseur aux rayons, sous la forme d'une répartition d'intensité gaussienne selon la normale, et on effectue une approximation paraxiale dans un système local de coordonnées lié à chaque rayon [14]. En pratique, on est amené à résoudre le système classique (5) et (6) pour calculer la trajectoire moyenne du faisceau, auquel on associe deux autres équations qui permettent la détermination de la largeur du faisceau et de la courbure du front de phase [15].

$$
\begin{aligned}
& \frac{d q}{d s}=c(s) p(s) \\
& \frac{d p}{d s}=-\frac{1}{c^{2}} \frac{\partial^{2} c}{\partial n^{2}} q(s)
\end{aligned}
$$

où $\mathbf{n}$ est l'abscisse curviligne selon la normale au rayon. L'évolution du faisceau est donnée par :

$$
p(s, n)=A \sqrt{c / r q} \exp \left[-i \omega\left(\tau+0,5(p / q) n^{2}\right)\right]
$$

où $A$ est une constante arbitraire et $\tau$ le temps de trajet le long du rayon. Le champ total est reconstitué par sommation des contributions des différents faisceaux résultant de la décomposition de la source et passant au voisinage du récepteur.

Parmi les avantages de cette technique, citons la prise en compte de la diffraction, donc l'absence de problème aux caustiques, le fait qu'il n'est pas nécessaire de calculer les rayons propres et qu'un code de sommation de faisceaux gaussiens peut être aussi rapide qu'un code de tracé de rayons classique. La méthode étant d'application récente en acoustique aérienne, il est difficile d'en cerner les limitations, mais il faut se rappeler qu'il s'agit d'une approche haute fréquence et qu'on doit s'attendre à des difficultés pour obtenir le champ très à l'intérieur des zones d'ombre.

\section{INFLUENCE DE LA TURBULENCE}

La présence des fluctuations turbulentes de l'atmosphère se manifeste essentiellement par deux effets : réduction des interférences due à la décorrélation partielle entre trajet direct et trajet réfléchi ; remontée du niveau dans les zones d'ombre par diffusion du son hors de la direction moyenne de propagation.

\section{Méthode des moments}

Cette méthode, maintenant classique, consiste à former des équations moyennées pour les moments successifs du champ : onde cohérente, corrélations spatiales, fluctuations d'intensité. La turbulence diffusant l'énergie dans un cône d'angle faible autour de la direction de propagation initiale, l'équation du départ est l'E.P.S. A titre d'exemple nous donnons l'équation régissant l'évolution du moment d'ordre 1 :

$$
2 i k_{0} \frac{\partial}{\partial x}\langle\psi\rangle+\Delta_{1}\langle\psi\rangle+2 k_{0}^{2}\langle\epsilon \psi\rangle=0
$$


où $\epsilon$ est la fluctuation d'indice : $\epsilon=-\left(T^{\prime} / 2 T_{0}+v^{\prime} / c_{0}\right)$ et $\Delta_{\perp}$ désigne le Laplacien dans le plan perpendiculaire à $\mathrm{x}$. On illustre ainsi le problème de fermeture inhérent à cette approche : il est nécessaire de faire une hypothèse a priori sur le champ $\in$ pour exprimer le terme $\langle\in \psi\rangle$. A l'aide de l'hypothèse de corrélation microscopique selon $x$ il devient possible de déterminer les différentes quantités statistiques intéressantes et la comparaison avec les résultats expérimentaux est très bonne au moins pour les deux premiers moments ; la prédiction des fluctuations d'intensité et notamment de leur saturation reste cependant très perfectible. D'autre part la prise en compte d'un milieu moyen inhomogène où coexistent plusieurs trajets partiellement corrélés ne semble pas avoir été réalisée actuellement.

\section{Simulations numériaues directes}

Afin de répondre à ce dernier point, tout en s'affranchissant de l'hypothèse ad hoc de corrélation microscopique, une nouvelle approche a été développée récemment. La turbulence est considérée comme une suite de réalisations indépendantes d'un champ aléatoire figé. Pour chaque réalisation le calcul de la propagation peut être effectué selon une technique déterministe classique (rayons, EPS). Les grandeurs statistiques sont ensuite obtenues par moyenne d'ensemble sur un grand nombre de réalisations (une centaine par exemple). Actuellement de tels calculs ont été réalisés dans le cas d'une turbulence simple homogène et isotrope en dimension 2 ou 3. Les résultats obtenus sont très encourageants, notamment en ce qui concerne les fluctuations d'intensité et leur évolution avec les échelles turbulentes et la fréquence de l'onde. Quelques études ont également été réalisées en présence d'un sol, dans des conditions similaires à celles de la référence [2], et reproduisent de façon convaincante les résultats expérimentaux sur l'évolution du niveau aux creux d'interférence. Le seul reproche que l'on puisse faire à la méthode est la lourdeur des calculs et ceci limite pour l'instant son application à des configurations test de façon à définir des tendances.

\section{CONCLUSION}

De ce panorama rapide et forcément incomplet des méthodes de calcul utilisées en acoustique aérienne il ressort qu'il n'existe actuellement aucune méthode universelle qui permettrait de prendre en compte tous les phénomènes physiques mis en jeu : réfraction par les gradients, diffraction par des écrans ou obstacles, diffusion par la turbulence. L'usage conjoint d'au moins deux méthodes complémentaires s'impose, par exemple rayons et équation parabolique. Cependant, les progrès réalisés dans les traitements théoriques et numériques permettent l'analyse de situations complexes où aspects déterministes et aléatoires coexistent.

\section{REFERENCES}

[1] ATTENBOROUGH, K.A., 1988 : Review of ground effects on outdoor sound propagation from continuous broadband sources. Applied Acoustics 24, p. 289-319.

[2] DAIGLE, G.A., PIERCY, J.E., EMBLETON T.F.W., 1978 : Effect of atmospheric turbulence on the interference of sound waves near a hard boundary. JASA 64, p. 1505-1513.

[3] TATARSKI, V.I., 1971 : The effects of turbulent atmosphere on wave propagation. IPST Keter Press, Jerusalem.

[4] MARTIN, J.M., FLATTE, S.M., 1988 : Intensity images and statistics from numerical simulation of wave propagation in 3-D random media. Applied Optics 27, p. 2111-2126.

[5] KARWEIT, M.J., BLANC-BENON, Ph., JUVE, D., COMTE-BELLOT, G., 1991 : Simulation of the propagation of an acoustic wave through a turbulent velocity field. A study of phase variance. JASA 89, p. 52-62.

[6] JUVE, D., BLANC-BENON Ph., COMTE-BELLOT, G., 1991 : Transmission of acoustic waves through mixing layers and 2D isotropic turbulence. In "turbulence and coherent structures" Ed. $\mathrm{O}$. Metais and M. Lesieur Kluwer Academis Published, Dordrecht. 
[7] DINAPOLI, F.R., DEAVENPORT, R.L., 1979 : Numerical models of underwater acoustic propagation in "ocean acoustics", Ed. J.A. Desanto , Topics in current physics, Springer Verlag, Berlin.

[8] RASPET, R. \& al., 1985 : A fast field program for sound propagation in a layered atmosphere above an impedance ground.JASA 77, p. 345-352.

[9] FRANKE, S.F., RASPET, R., LIU, C.H., 1988 : Numerical predictions of atmospheric soundpressure levels in shadow zones. JASA 83, p. 816-820.

[10] CANDEL, S.M., 1977 : Numerical solution of conservation equations arising in linear wave theory : application to aeroacoustics. J. Fluid Mech. 83, p. 465-493.

[11] TAPPERT, F.D., 1977 : The parabolic approximation method. In "wave propagation and underwater acoustics". Ed. J.B. Keller \& J.S. Papadakis. Lecture Notes in Physics 70, Springer Verlag, Berlin.

[12] CLAERBOUT, J.F., 1970 : Coarse grid calculations of waves in inhomogeneous media with application to the delineation of complicated seismic structures. Geophysics 35, p. 407-418.

[13] ST.MARY, D.F., DING LEE, 1985 : Analysis of an implicit finite difference solution to an underwater wave propagation problem. J. Comp. Phys. 57, p. 378-390.

[14] CERVENY, V., POPOV, M.M., PSENCIK, I., 1982 : Computation of wave fields in inhomogeneous media - Gaussian beam approach. Geophys. J.R. Astr. Soc. 70, p. 109-128.

[15] PORTER, M.B., BUCKER, H.P., 1987 : Gaussian beam tracing for computing ocean acoustic fields. JASA 82, p. 1349-1359.

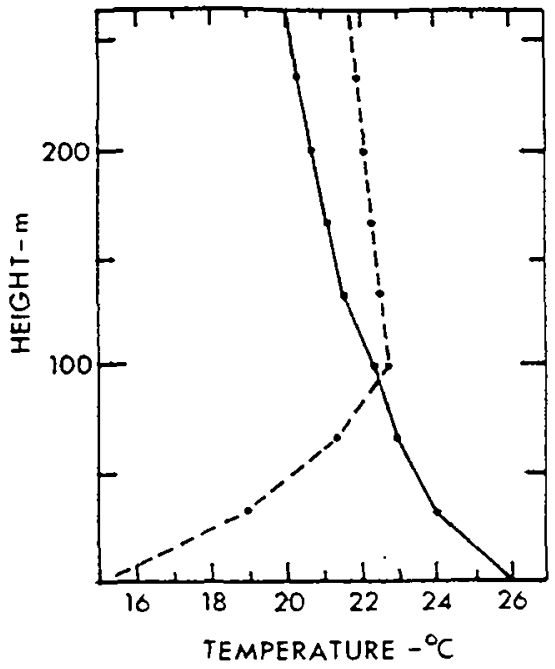

Figure 1 :

Exemples d'évolution de la température avec altitude

( $\rightarrow$ - conditions d'inversion) 
Tableau I : Différentes valeurs de hauteur de rugosité en fonction du terrain

\begin{tabular}{|l|c|}
\hline NATURE DU TERRAIN & HAUTEUR DE RUGOSITE (m) \\
\hline eau, glasse lisse & $10^{-4}$ \\
\hline herbe rase & $10^{-2}$ \\
\hline pâturage & 0,2 \\
\hline banlieue & 0,6 \\
\hline forêt, ville & 1 à 5 \\
\hline
\end{tabular}

Tableau II: Paramètres turbulents relevés lors des expériences décrites dans la référence [2]. $\sigma$ valeur efficace des fluctuations ; L échelle de corrélation. Les valeurs entre parenthèses désignent les écart-types des mesures pour chaque jour.

\begin{tabular}{|c|l||c|c|c|c|}
\hline Day & Description & $\begin{array}{c}\sigma_{\mathrm{v}} \\
(\mathrm{m} / \mathrm{s})\end{array}$ & $\begin{array}{c}\sigma_{\mathrm{T}} \\
\left({ }^{\circ} \mathrm{C}\right)\end{array}$ & $\begin{array}{c}\mathrm{L}_{\mathrm{v}} \\
(\mathrm{m})\end{array}$ & $\begin{array}{c}\mathrm{L}_{\mathrm{T}} \\
(\mathrm{m})\end{array}$ \\
\hline 1 & None recorded & 0,7 & 0,8 & 0,5 & 0,9 \\
& & $(0.1)$ & $(0.3)$ & $(0,1)$ & $(0,2)$ \\
\hline 2 & Bright sunny day, no clouds & 1,1 & 1,1 & 1,2 & 1,3 \\
& & $(0,2)$ & $(0,2)$ & $(0,2)$ & $(0,2)$ \\
\hline 3 & Hazy sun, 30\% thin clouds, & 0,9 & 0,7 & 1,1 & 1,7 \\
& high winds & $(0,2)$ & $(0,1)$ & $(0,3)$ & $(0,7)$ \\
\hline 4 & Clear and sunny, no clouds & 0,5 & 0,9 & 0,5 & 1,0 \\
& & $(0,1)$ & $(0,2)$ & $(0,1)$ & $(0,2)$ \\
\hline 5 & Evening, no clouds, light & 0,2 & 0,7 & 0,5 & $\cdots$ \\
& winds & $(0,1)$ & $(0,6)$ & $(0,1)$ & \\
\hline 6 & Sunny with variable clouds & 0,8 & 0,4 & 1,2 & 1,2 \\
& (overcast) stiff SW breeze & $(0,2)$ & $(0,1)$ & $(0,2)$ & $(0,5)$ \\
& & & & & \\
\hline
\end{tabular}

